\title{
A de novo heterozygous variant in KAT6A is associated with a newly named neurodevelopmental disorder Arboleda-Tham syndrome-a case report
}

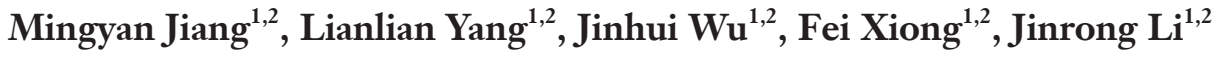 \\ ${ }^{1}$ Department of Pediatrics, West China Second University Hospital, Sichuan University, Chengdu, China; ${ }^{2}$ Ministry of Education Key Laboratory of \\ Birth Defects and Related Diseases of Women and Children, Sichuan University, Chengdu, China \\ Correspondence to: Jinrong Li. No. 20, Section 3, Renmin South Road, Chengdu, China. Email: ljinrong224@yeah.net.
}

\begin{abstract}
Arboleda-Tham syndrome (OMIM\#616268) is a newly named neurodevelopmental disorder, which is an autosomal dominant hereditary disease characterized by genetic variants. The clinical manifestations include global developmental delay, primary microcephaly, and craniofacial dysmorphism, as well as more varied features such as feeding difficulties, cardiac defects, and ocular anomalies. Currently, due to restricted knowledge of Arboleda-Tham syndrome and less specific pathological manifestations, it is difficult to diagnose at the early stages of the disease. Here, we present a case with obvious growth retardation and intellectual disability, accompanied by other manifestations including dysmorphic features of the ears, facial dysmorphism, right cryptorchidism, and inguinal hernia. Routine laboratory tests including blood-urine tandem mass spectrometry, urine gas chromatographic mass spectrometry, karyotype, echocardiography, automatic auditory brainstem responses, serum levels of calcium, phosphorus, vitamin $\mathrm{D}$, creatine kinase $(\mathrm{CK})$, and $\mathrm{CK}$ isoenzyme (CK-MB), and brain magnetic resonance imaging showed negative results. A de novo heterozygous variant in $K A T 6 A$, c.57delA (p.Val20*), was detected by trio-based whole exome sequencing and subsequent validation by Sanger sequencing in the patient, which was absent in both the parents. The patient received rehabilitation and nutritional intervention. The testis reduction and orchiopexy was scheduled when he was 1 year old. Our report extends the phenotype-genotype map of Arboleda-Tham syndrome, and also expands the mutant spectrum of the KAT6A gene. Moreover, this case emphasizes the timely conduction of whole exome sequencing for the early diagnosis of Arboleda-Tham syndrome, and spares patients from meaningless examinations and ineffective treatments.
\end{abstract}

Keywords: KAT6A; neurodevelopmental disease; clinical genetics; whole exome sequencing; case report

Submitted Mar 31, 2021. Accepted for publication May 26, 2021.

doi: $10.21037 / \mathrm{tp}-21-206$

View this article at: http://dx.doi.org/10.21037/tp-21-206

\section{Introduction}

Among all cases of intellectual disability or global developmental delay, it is estimated that copy-number variations $(\mathrm{CNVs})$ and $\mathrm{X}$-linked and autosomal-recessive inherited conditions are responsible for $25 \%$. The novel heterozygous monogenic variants are the main cause of mental retardation. By using DNA sequencing technology, the pathogeny of $40 \%$ of severe mental retardation has been revealed $(1,2)$. Arboleda-Tham syndrome (OMIM\#616268) is a newly neurodevelopmental disorder, caused by variants in the KAT6A gene (also known as MOZ, MYST3; NM_006766.5) located on chromosome $8 \mathrm{q} 11.21$. The $K A T 6 A$ gene, codes for one member of the histone acetyltransferase (HAT) family MYST. Collectively, HAT and histone deacetylase (HDAC) enzymes play key roles in essential cellular processes including the cell cycle, metabolism, apoptosis, and stem cell maintenance through remodeling chromatin $(3,4)$. The transcription factors Runx1 and Runx2 are directly regulated by the KAT6A gene by its serine- and methionine-rich domain $(5,6)$. 

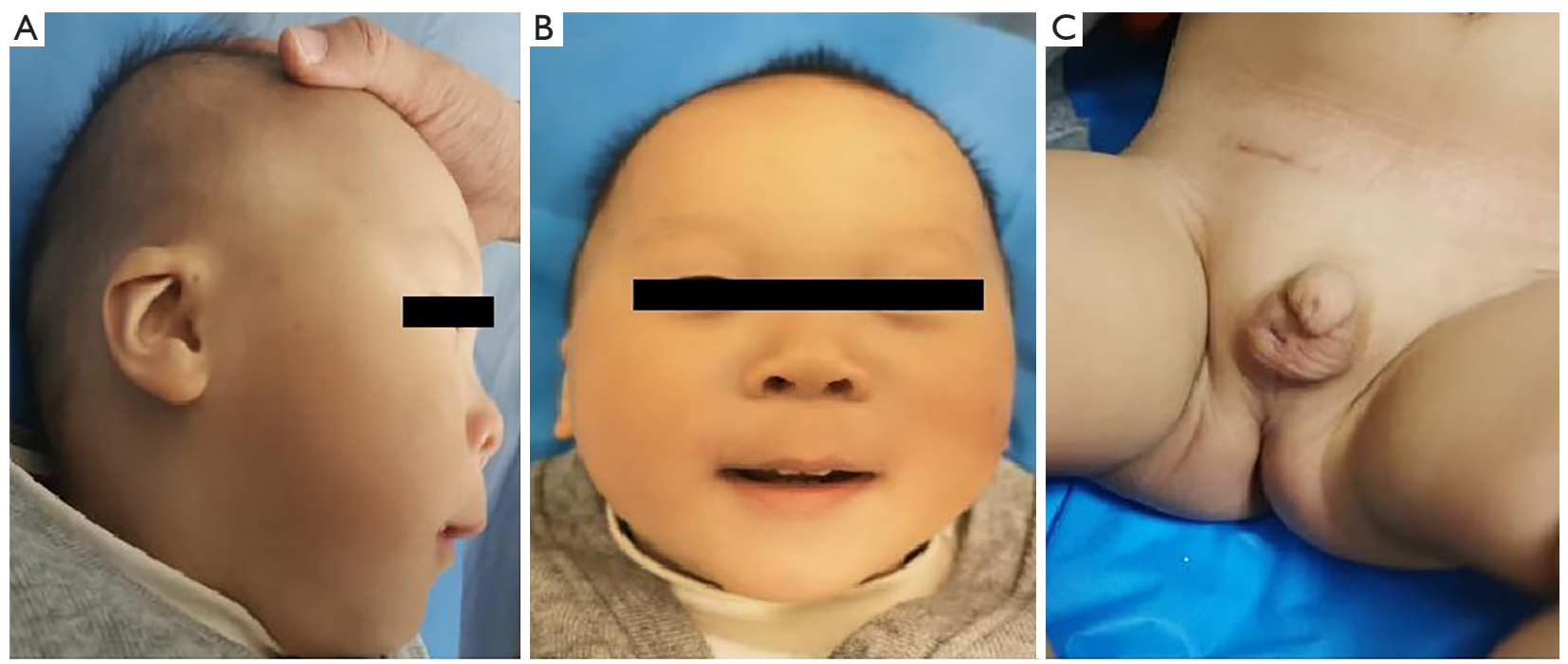

Figure 1 Clinical features of the patient. (A) Lateral auricle collapse and binaural medial fistula. (B) Thin upper lip and large forehead. (C) Right oblique inguinal hernia and right cryptorchidism.

To our knowledge, a total of 85 similar cases and gene studies of Arboleda-Tham syndrome have been reported to date since it was first recognized in 2015 by Arboleda et al. (1-24). These probands have common clinical manifestations such as global developmental delay, including severe speech disability, significant craniofacial dysmorphism, and primary microcephaly, as well as more varied concomitant features including feeding problems, ocular anomalies, and cardiac defects which provide a suspicion of this disease and finally diagnosed by the gene analysis (7). Herein, we report an additional ArboledaTham syndrome case with a new clinical phenotype and genotype. In addition, we summarized the research of the phenotypic and genetic spectrum from the reported KAT6A cases.

We present the following article in accordance with the CARE reporting checklist (available at http://dx.doi. org/10.21037/tp-21-206).

\section{Case presentation}

The patient was a boy aged 1 year 3 months who was admitted to our hospital due to obvious growth retardation and intellectual disability for 1 year. His parents noticed these problems including deteriorating growth retardation, intellectual disability with medium speech impairment, motor delay, cryptorchidism, and inguinal hernia. The boy was born to a nonconsanguineous couple, and the mother was a thalassemia gene carrier. During the third trimester, the proband's mother suffered from cholestasis and gestational diabetes.

He was delivered at 38 weeks by cesarean section, with Apgar scores of 9/10/10 at 1, 5, and $10 \mathrm{~min}$, respectively. The birth weight of the child was $2,680 \mathrm{~g}\left(\mathrm{P}_{3}-\mathrm{P}_{10},-1 \mathrm{SD}\right.$ to $-2 \mathrm{SD})$ and height $50 \mathrm{~cm}\left(\mathrm{P}_{25}-\mathrm{P}_{50}, 0 \mathrm{SD}\right.$ to $\left.-1 \mathrm{SD}\right)$. When he reached 1 year 3 months old, his weight was $9.35 \mathrm{~kg}$ $\left(\mathrm{P}_{10}-\mathrm{P}_{20},-1.11 \mathrm{SD}\right)$, height $75.4 \mathrm{~cm}\left(\mathrm{P}_{5}-\mathrm{P}_{10},-1.49 \mathrm{SD}\right)$, and cranial circumference $44.5 \mathrm{~cm}\left(\mathrm{P}_{0}-\mathrm{P}_{3},-1.92 \mathrm{SD}\right)$. At 15 months old, his weight was $9.35 \mathrm{~kg}$, length was $75.4 \mathrm{~cm}$, and the occipitofrontal circumference (OFC) was $44.5 \mathrm{~cm}$. The boy showed some evident abnormal features such as dysmorphic features of the ears (lateral auricle collapse, binaural medial fistula), facial dysmorphism (thin upper lip, large forehead), right cryptorchidism, and inguinal hernia (Figure 1). Routine test results for serum alkaline phosphatase (ALP) increased to 1,700 IU/L (reference value: 140-420 IU/L), while the levels of his serum calcium, phosphorus, vitamin $\mathrm{D}$, creatine kinase (CK), and CK isoenzyme (CK-MB) were all normal. The karyotype analysis was $46, \mathrm{XY}$. His skeletal X-ray imaging, brain MRI, and AABR (automated auditory brainstem response) were normal.

All procedures performed in studies involving human participants were in accordance with the ethical standards of the institutional and/or national research committee(s) and with the Helsinki Declaration (as revised in 2013). Written 
informed consent was obtained from his parents.

\section{Developmental assessment}

The child displayed abnormal parameters of growth indicators such as raising his head at 4 months of age, rollover at more than 7 months of age, sitting without help at 1 year of age, and did not speak any meaningful words, such as papa and mama, for communication. His DQ scores of language, adaptation, gross motor, fine motor, and personal social functioning on the Gesell developmental scales were $65,75,70,80$, and 75 respectively, revealing that speech impairment was the prominent disability.

\section{Molecular findings}

DNA was extracted from peripheral blood samples, and WES was performed. Sanger sequencing was used to verify the mutations in the proband, and the variant was classified following the 2015 guidelines of the American College of Medical Genetics (ACMG). A normal karyotype (46, XY) was determined, indicating no relevant CNVs to explain the proband's clinical features. A novel variant, c.57delA, was detected in $K A T 6 A$ of the proband, which is a nonsense mutation leading to an amino acid change, p.Val20*. There were no relevant variants in the parents' genotypes, which were normal (Figure 2). The de novo variant in the proband and his parents was detected by Sanger sequencing and met the criteria to be identified as a pathogenic variant: PVS1+PS2+PM2 according to the 2015 ACMG guidelines. This new variant has never been previously described in the databases of genes.

\section{Diagnosis and treatment}

The patient was initially diagnosed with cryptorchidism and received the testis reduction and orchiopexy when he was 1 year old. Then based on the clinical manifestations and genetic mutation, the diagnosis of Arboleda-Tham syndrome was clear. Arboleda-Tham syndrome is a newly identified neurodevelopmental disorder caused by variants in the $K A T 6 A$ gene. Then the patient received rehabilitation and nutritional intervention, and was followed up regularly for every 3 months. After nutritional intervention, his physical growth is normal, and the testicle is located in the scrotum after surgery. With rehabilitation training, he can say dad, mum, grandfather, grandmother and other simple words. But there is no targeted therapy for this disease at present.

\section{Discussion}

The clinical features noticed in our patient which were characteristic of Arboleda-Tham syndrome including developmental delay, speech delay, microcephaly, lateral auricle collapse, binaural medial fistula, large forehead, thin upper lip, right oblique inguinal hernia, and right cryptorchidism (8-13). Notably, the binaural medial fistula and lateral auricle collapse observed in our patient had never been reported previously. Contrary to other reported cases, our patient had an abnormally high level of serum ALP when he was 10 months old. Based on the research about KAT6A regulation of the bone marrow-derived mesenchymal stem cells through Nrf2/ARE signaling pathway, we inferred that the level of the ALP may also be influenced by this way. But existence of abnormal level of serum phosphatase alkaline in this patient may also be coincidental and further research is needed to confirm this potential relationship (14).

All previously reported cases of Arboleda-Tham syndrome had intellectual disability, global developmental delay, and craniofacial dysmorphism. Among the various craniofacial dysmorphisms, bulbose or broad nasal tip was the most common (85\%). Others include eye abnormalities (proptosis, palpebral ptosis, hypertelorism, deep set, and strabismus), ear anomalies (large, low set, rotated, small earlobe), thin upper lip, bitemporal narrowing, microcephaly, frontal bossing, large forehead, and micrognathia. Some of the clinical manifestations including microcephaly, craniofacial dysmorphism, and cardiac defects may be found by the ultrasound exam at the fetal period and the prenatal genetic analysis would be conducted under the guidance of multidisciplinary team (MDT). Notably, among the reported ear abnormalities, the binaural medial fistula and lateral auricle collapse observed in our patient have never been mentioned. This new clinical manifestation in ArboledaTham syndrome especially extended phenotype of ear. However, speech delay or absent speech were observed in $99 \%$ of the subjects. Neonatal hypotonia occurred in $62.0 \%$ of the subjects, and abnormal gait was identified in $67 \%$ of the subjects when they started walking. Other manifestations mainly include seizures, congenital heart disease, and recurrent infections (Table 1) (3-5,7,13,17-25). Recurrent infections have been observed in $52 \%$ of reported patients, among which $71 \%$ of subjects carry late 


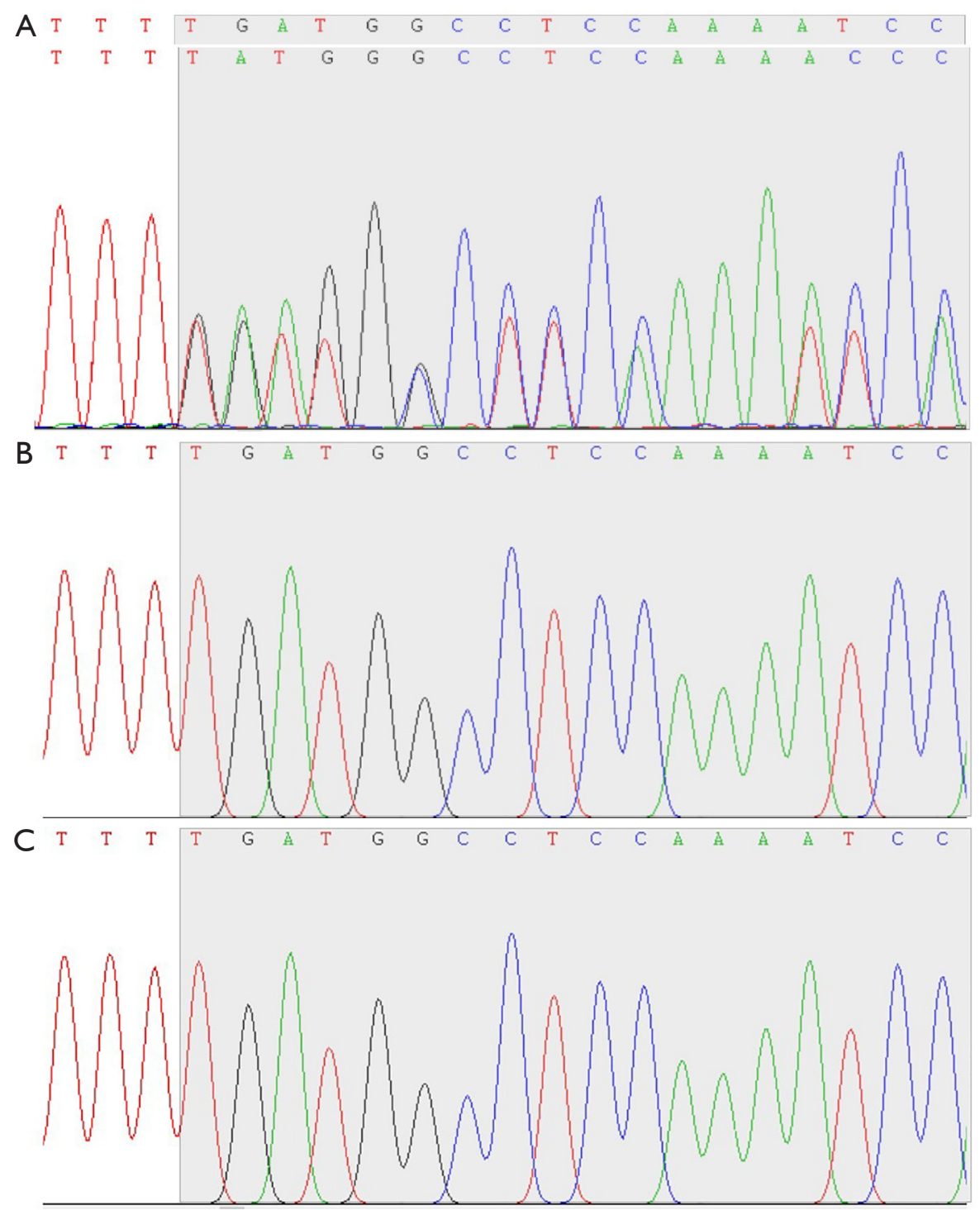

Figure 2 Comparative analysis of $K A T 6 A$ sequences in the child and his parents. (A) A heterozygous variant, c.57delA, in $K A T 6 A$ was detected in the child. (B) No variant in $K A T 6 A$ was detected in the mother. (C) No variant in $K A T 6 A$ was detected in the father.

truncating variants (7). This finding is consistent with the theory that $K A T 6 A$ plays an essential role in the long-term repopulation of hematopoietic stem cells (8). The study from Good-Jacobson et al. demonstrated that KAT6A is of great importance in lymphocyte differentiation. Their study showed that the absence of KAT6A resulted in significant impairments in the ability of germinal center B cells to generate dark zone centroblasts, and damaged both BCL-6 expression and cell-cycle progression (9). In this way, mutations of the KAT6A gene lead to impairments in immune functions, with increased susceptibility to recurrent infection.

The boy was born to a nonconsanguineous couple, and the mother was a thalassemia gene carrier. The KAT6A gene, also named MOZ or MYST3 and located on chromosome $8 \mathrm{q} 11.21$, was known earlier as a fusion protein in patients with acute myelogenous leukemia (10). While the thalassemia gene is located on $11 \mathrm{p} 15.4$. There is no correlation between them by reviewing the researches before. KAT6A is found in the majority of tissues in the human protein atlas including the brain and heart. The gene consists of 18 exons ( 16 coding), and encodes a 
Table 1 Clinical features of Arboleda-Tham syndrome reported to date

\begin{tabular}{|c|c|c|c|c|c|c|}
\hline Clinical features & $\begin{array}{l}\text { Urreizti et al. } \\
\qquad \text { [2020] }\end{array}$ & $\begin{array}{l}\text { Kennedy et al. } \\
\text { [2019] }\end{array}$ & $\begin{array}{l}\text { Alkhateeb et al. } \\
\qquad[2019]\end{array}$ & $\begin{array}{l}\text { Efthymiou et al. } \\
\qquad[2018]\end{array}$ & Trinh et al. [2018] & $\begin{array}{l}\text { Present study } \\
\text { [2021] }\end{array}$ \\
\hline $\begin{array}{l}\text { Developmental } \\
\text { delay/intellectual disability }\end{array}$ & + & + & + & + & + & + \\
\hline Microcephaly & + & + & & + & + & + \\
\hline Speech delay & + & + & + & + & + & + \\
\hline Abnormal gait & + & + & + & + & & \\
\hline Autistic behavior & + & + & & & & \\
\hline Facial dysmorphism & + & + & + & + & + & + \\
\hline Large forehead & + & + & & + & & \\
\hline Broad nasal tip & + & + & & + & + & \\
\hline Thin upper lip & & & + & & + & + \\
\hline \multicolumn{7}{|l|}{ Other features } \\
\hline Seizures & + & + & & + & + & \\
\hline Congenital heart defect & + & + & & & & \\
\hline Recurrent infections & + & + & & & & \\
\hline
\end{tabular}

+ indicate: positive signs.

member of the HAT family, MYST. The HAT complex acetylates lysine-9 residues in histone $\mathrm{H} 3$ (H3K9), which is related to transcriptionally active genes. As previous studies have demonstrated, histone deacetylation is associated with transcriptional silencing. In this way, the KAT6A gene is involved in the regulation of a multitude of genes, which play essential roles during the progression of human growth and development (11). There are 5 functional domains in the KAT6A gene: a double-plant homeodomain finger that binds to acetylated histone $\mathrm{H} 3$ tails, a nuclear localization domain, a histone-acetyl-transferase domain, an acidic glutamate/aspartate-rich region, and a serine/methioninerich region that can directly bind and regulate the transcription factors Runx1 and Runx2 (11-13) (Figure 3). Previous studies have demonstrated that in rodents, KAT6A is expressed in most tissues from embryos to adult mice, and is significantly important for the development of the skeleton, the hematopoietic system, and the thymus (11-13). Voss et al. reported that inserting a neo coding sequence into exon 16 in a KAT6A gene homozygous knockout mouse model resulted in severe detriments including craniofacial and heart abnormalities as well as normal hematopoietic cells (11). Moreover, the research showed that mice heterozygous for C-terminal deletion displayed an intermediate phenotype compared to the homozygous and wild-type mice regarding the number and function of hematopoietic progenitor cells. Further analysis of Hox gene expression demonstrated that the effect of the KAT6A gene was dose-dependent (8,11-13). These genetic analyses were reinforced with other relevant research in zebrafish illustrating that $K A T 6 A$ is essential to the renewal of neural stem cells and the normal development of pharyngeal segments (8).

In conclusion, we described an additional case of an emerging and distinctive disorder, Arboleda-Tham syndrome, and revealed a novel variant in KAT6A. The similar clinical manifestations of our patient with reported patients with Arboleda-Tham syndrome and classification as "pathogenic" by ACMG of the novel KAT6A variant, lead us to hypothesize that the novel $K A T 6 A$ variant is responsible for the Arboleda-Tham syndrome phenotype in our patient. Still, further functional study of the variant is needed in the 


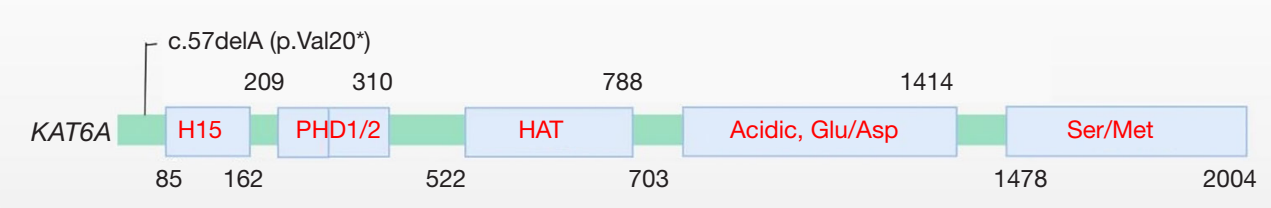

Figure 3 A schematic illustration of the main functional units of human KAT6A. KAT6A (also known as MOZ, MYST3; NM_006766.5): the nuclear localization domain (H15; AA 85-162), a double-plant homeodomain finger (PHD; AA 209-310), a histone-acetyl-transferase domain (HAT; AA 522-703), an acidic glutamate/aspartate-rich domain (AA 788-1414), and a serine/methionine-rich region (AA 14782004). A novel variant, c.57delA, was detected in $K A T 6 A$ of the proband, which is a nonsense mutation that causes an amino acid change, p.Valr20*.

future. Also, given the severity and magnitude of its multisystem detriments, we emphasize the importance of early diagnosis of Arboleda-Tham syndrome. We suggest that timely intervention with WES is crucial and may help spare patients from unnecessary diagnostic examinations with further ineffective and even harmful treatments.

Our report refines the genotype-phenotype map of Arboleda-Tham syndrome and expands the mutant spectrum of the KAT6A gene.

\section{Acknowledgments}

The authors thank the family members of the patient for providing blood samples, co-operating with therapeutic strategies, and agreeing to participate in this study.

Funding: Youth Innovation Project of Medical Research in Sichuan Province (Q18015). Recipient: Jinrong Li.

\section{Footnote}

Reporting Checklist: The authors have completed the CARE reporting checklist. Available at http://dx.doi.org/10.21037/ tp-21-206

Conflicts of Interest: All authors have completed the ICMJE uniform disclosure form (available at http://dx.doi. org/10.21037/tp-21-206). The authors have no conflicts of interest to declare.

Ethical Statement: The authors are accountable for all aspects of the work in ensuring that questions related to the accuracy or integrity of any part of the work are appropriately investigated and resolved. All procedures performed in studies involving human participants were in accordance with the ethical standards of the institutional and/or national research committee(s) and with the Helsinki
Declaration (as revised in 2013). Written informed consent was obtained from his parents.

Open Access Statement: This is an Open Access article distributed in accordance with the Creative Commons Attribution-NonCommercial-NoDerivs 4.0 International License (CC BY-NC-ND 4.0), which permits the noncommercial replication and distribution of the article with the strict proviso that no changes or edits are made and the original work is properly cited (including links to both the formal publication through the relevant DOI and the license). See: https://creativecommons.org/licenses/by-nc-nd/4.0/.

\section{References}

1. Gilissen C, Hehir-Kwa JY, Thung DT, et al. Genome sequencing identifies major causes of severe intellectual disability. Nature 2014;511:344-7.

2. Moeschler JB, Shevell M, Committee on Genetics. Comprehensive evaluation of the child with intellectual disability or global developmental delays. Pediatrics 2014;134:e903-18.

3. Urreizti R, Lopez-Martin E, Martinez-Monseny A, et al. Five new cases of syndromic intellectual disability due to KAT6A mutations: widening the molecular and clinical spectrum. Orphanet J Rare Dis 2020;15:44.

4. Arboleda VA, Lee H, Dorrani N, et al. De novo nonsense mutations in KAT6A, a lysine acetyl-transferase gene, cause a syndrome including microcephaly and global developmental delay. Am J Hum Genet 2015;96:498-506.

5. Tham E, Lindstrand A, Santani A, et al. Dominant mutations in KAT6A cause intellectual disability with recognizable syndromic features. Am J Hum Genet 2015;96:507-13.

6. Pelletier N, Champagne N, Stifani S, et al. MOZ and MORF histone acetyltransferases interact with the 
Runt-domain transcription factor Runx2. Oncogene 2002;21:2729-40.

7. Kennedy J, Goudie D, Blair E, et al. KAT6A syndrome: genotype-phenotype correlation in 76 patients with pathogenic KAT6A variants. Genet Med 2019;21:850-60.

8. Katsumoto T, Aikawa Y, Iwama A, et al. MOZ is essential for maintenance of hematopoietic stem cells. Genes Dev 2006;20:1321-30.

9. Good-Jacobson KL, Chen Y, Voss AK, et al. Regulation of germinal center responses and B-cell memory by the chromatin modifier MOZ. Proc Natl Acad Sci USA 2014;111:9585-90.

10. Borrow J, Stanton VP, Andresen JM, et al. The translocation $\mathrm{t}(8 ; 16)(\mathrm{p} 11 ; \mathrm{p} 13)$ of acute myeloid leukaemia fuses a putative acetyltransferase to the CREB-binding protein. Nat Genet 1996;14:33-41.

11. Voss AK, Collin C, Dixon MP, et al. Moz and retinoic acid coordinately regulate $\mathrm{H} 3 \mathrm{~K} 9$ acetylation, Hox gene expression, and segment identity. Dev Cell 2009;17:674-86.

12. Klein BJ, Lalonde ME, Côté J, et al. Crosstalk between epigenetic readers regulates the MOZ/MORF HAT complexes. Epigenetics 2014;9:186-93.

13. Efthymiou S, Salpietro V, Bettencourt C, et al. Paroxysmal movement disorder and epilepsy caused by a de novo truncating mutation in KAT6A. J Pediatr Genet 2018;7:114-6.

14. Fei D, Wang Y, Zhai Q, et al. KAT6A regulates stemness of aging bone marrow-derived mesenchymal stem cells through Nrf2/ARE signaling pathway. Stem Cell Res Ther 2021;12:104.

15. Thomas T, Corcoran LM, Gugasyan R, et al. Monocytic leukemia zinc finger protein is essential for the development of long-term reconstituting hematopoietic stem cells. Genes Dev 2006;20:1175-86.

16. Miller CT, Maves L, Kimmel CB. moz regulates Hox expression and pharyngeal segmental identity in zebrafish.

Cite this article as: Jiang M, Yang L, Wu J, Xiong F, Li J. A de novo heterozygous variant in $K A T 6 A$ is associated with a newly named neurodevelopmental disorder Arboleda-Tham syndrome-a case report. Transl Pediatr 2021;10(6):17481754. doi: $10.21037 /$ tp-21-206
Development 2004;131:2443-61.

17. Crump JG, Swartz ME, Eberhart JK, et al. Mozdependent Hox expression controls segment-specific fate maps of skeletal precursors in the face. Development 2006;133:2661-9.

18. Millan F, Cho MT, Retterer K, et al. Whole exome sequencing reveals de novo pathogenic variants in KAT6A as a cause of a neurodevelopmental disorder. Am J Med Genet A 2016;170:1791-8.

19. Murray CR, Abel SN, McClure MB, et al. Novel causative variants in DYRK1A, KARS, and KAT6A associated with intellectual disability and additional phenotypic features. J Pediatr Genet 2017;6:77-83.

20. Elenius V, Lähdesmäki T, Hietala $M$, et al. Food allergy in a child with de novo KAT6A mutation. Clin Transl Allergy 2017;7:19.

21. Gauthier-Vasserot A, Thauvin-Robinet C, Bruel AL, et al. Application of whole-exome sequencing to unravel the molecular basis of undiagnosed syndromic congenital neutropenia with intellectual disability. Am J Med Genet A 2017;173:62-71.

22. Satoh C, Maekawa R, Kinoshita A, et al. Three brothers with a nonsense mutation in KAT6A caused by parental germline mosaicism. Hum Genome Var 2017;4:17045.

23. Zwaveling-Soonawala N, Maas SM, Alders M, et al. Variants in KAT6A and pituitary anomalies. Am J Med Genet A 2017;173:2562-5.

24. Trinh J, Hüning I, Yüksel Z, et al. A KAT6A variant in a family with autosomal dominantly inherited microcephaly and developmental delay. J Hum Genet 2018;63:997-1001.

25. Alkhateeb A, Alazaizeh W. A novel de novo frameshift mutation in KAT6A identified by whole exome sequencing. J Pediatr Genet 2019;8:10-4.

(English Language Editor: C. Betlazar-Maseh) 